\title{
Evaluación del riesgo volcánico ante una posible erupción del Volcán Callaqui, Chile
}

\author{
Manuel Araneda C.* \\ María Soledad Avendaño R.*
}

Recibido en agosto de 2012; aceptado en julio de 2013

\begin{abstract}
Ice mass evaluation of the most important glacial areas of Callaqui volcano was made using gravimetric profiles, direct aerial observation and through areal photography's analysis. The Callaqui volcano is located in the VIII Region of Chile, geographic coordinates $37^{\circ} 55^{\prime} 20^{\prime \prime} \mathrm{S}$ and $71^{\circ} 26^{\prime} 50^{\prime \prime} \mathrm{W}$. The volcanic eruption of Callaqui volcano can melt the ice cover, water flow through streams and river could damage in Pangue and Ralco hydroelectric power station, located near by the "BioBio" river valley, additionally an indeterminate number of houses and agriculture facilities and not less important human lives.

Result of the investigation has proved a total of $0.424168 \mathrm{~km}^{3}$ of ice volume plus $3 \mathrm{~m}$ of snow during winter season. Assuming $0.8 \mathrm{gr} / \mathrm{cm}^{3}$ density of the ice and $0.25 \mathrm{gr} / \mathrm{cm}^{3}$ for the snow, a total of $0.339334 \mathrm{~km}^{3}$ of water would be the flow from Callaqui volcano. The material cost due the volcanic eruption was evaluated in US\$2,346x $10^{6}$.

Finally is recommended a setting of a monitoring system with special equipment.
\end{abstract}

Key words: Gravimetry, glaciers, volcanic hazards.

\section{Resumen}

A partir de perfiles gravimétricos, observaciones aéreas y apoyo de fotografía areal, se realizó una cubicación de las masas de hielo de los glaciales más relevantes que componen el edificio volcánico Callaqui, ubicado en la región del Bio-Bio, cuyas coordenadas geográficas son: $37^{\circ} 55^{\prime} 20^{\prime}$ ' S y $71^{\circ} 26^{\prime} 50^{\prime}$ ' O, Chile.

Ante una posible erupción del volcán Callaqui, las masas de hielo calculadas fluirían por diferentes quebradas y ríos, ocasionando gravísimos daños en las centrales hidroeléctricas de Pangue y Ralco pertenecientes al complejo hidroeléctrico

* Consultores independientes, Santiago, Chile, correo electrónico: segmi@netexpress.cl 
del río Bio-Bio. Siendo las centrales hidroeléctricas las inversiones materiales de

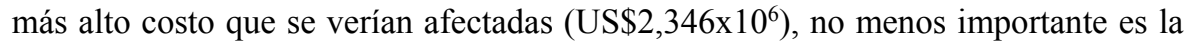
pérdida de vidas humanas, junto

a sus hogares, producción agrícola y otras obras menores difíciles de cuantificar.

El volumen total de hielo en los glaciares del volcán Callaqui que podrían fundirse en una posible erupción durante la temporada invernal es de $0.424168 \mathrm{~km}^{3}$ equivalentes a un volumen de agua de $0.339334 \mathrm{~km}^{3}$ el que se vería incrementado por la cubierta nival variable año a año, pudiendo ser de hasta tres metros, Araneda y Avendaño (1983), Araneda (1984).

Las densidades para calcular volúmenes de hielo y nieve, han sido estimadas en $0.8 \mathrm{gr} / \mathrm{cm}^{3}$ y $0.25 \mathrm{gr} / \mathrm{cm}^{3}$ respectivamente.

Finalmente se recomienda un sistema de monitoreo mediante una red sísmica telemétrica de cuatro estaciones junto a una red de inclinómetros y red geodésica para el control de movimientos verticales y desplazamientos horizontales.

Palabras clave: Gravimetría, glaciares, peligro volcánico.

\section{Introducción}

Durante el mes de mayo de 1984, el Departamento de Geología y Geofísica de la Universidad de Chile realizó una campaña geológica-geofísica en la VIII Región (Bio-Bio, Chile), donde se ubica el volcán Callaqui (Figura 1).

El volcán Callaqui, activo con fumarolas permanentes ha sido estudiado en forma parcial por Marangunic y Moreno (1981), Moreno et al. (1984). En esta oportunidad el tema tratado está relacionado con la determinación de los volúmenes de hielo permanente de los diferentes glaciares que componen el complejo del volcán Callaqui. Su cubicación se realizó mediante perfiles gravimétricos, fotografías aéreas y de una observación directa a través de un reconocimiento aéreo de helicóptero. La evaluación estuvo dirigida a cuantificar la masa de hielo y nieve del volcán Callaqui que podrían verse involucradas en una posible erupción. La rápida fusión de los glaciares y de la cubierta de nieve puede liberar volúmenes de agua suficientes para causar lahares potencialmente dañinos, siendo las vías de evacuación más probables aquella que proporciona la topografía, quebradas y ríos afluentes del río Bio-Bio en el cual están proyectadas las centrales hidroeléctricas que nos preocupan. El riesgo mayor podría considerarse entre los meses de junio a octubre por el volumen de hielo y nieve acumulados, principalmente en el glaciar más desarrollado que está ubicado en el flanco sur oriental del volcán Callaqui.

Otras consideraciones relevantes que podrían incidir en un mayor volumen de materiales que podrían descender desde la cumbre del volcán, pueden ser avalanchas de nieves activadas por el flujo termal en sectores adyacentes al centro eruptivo pudiéndose acentuar si la frecuencia e intensidad de los eventos sísmicos van en 


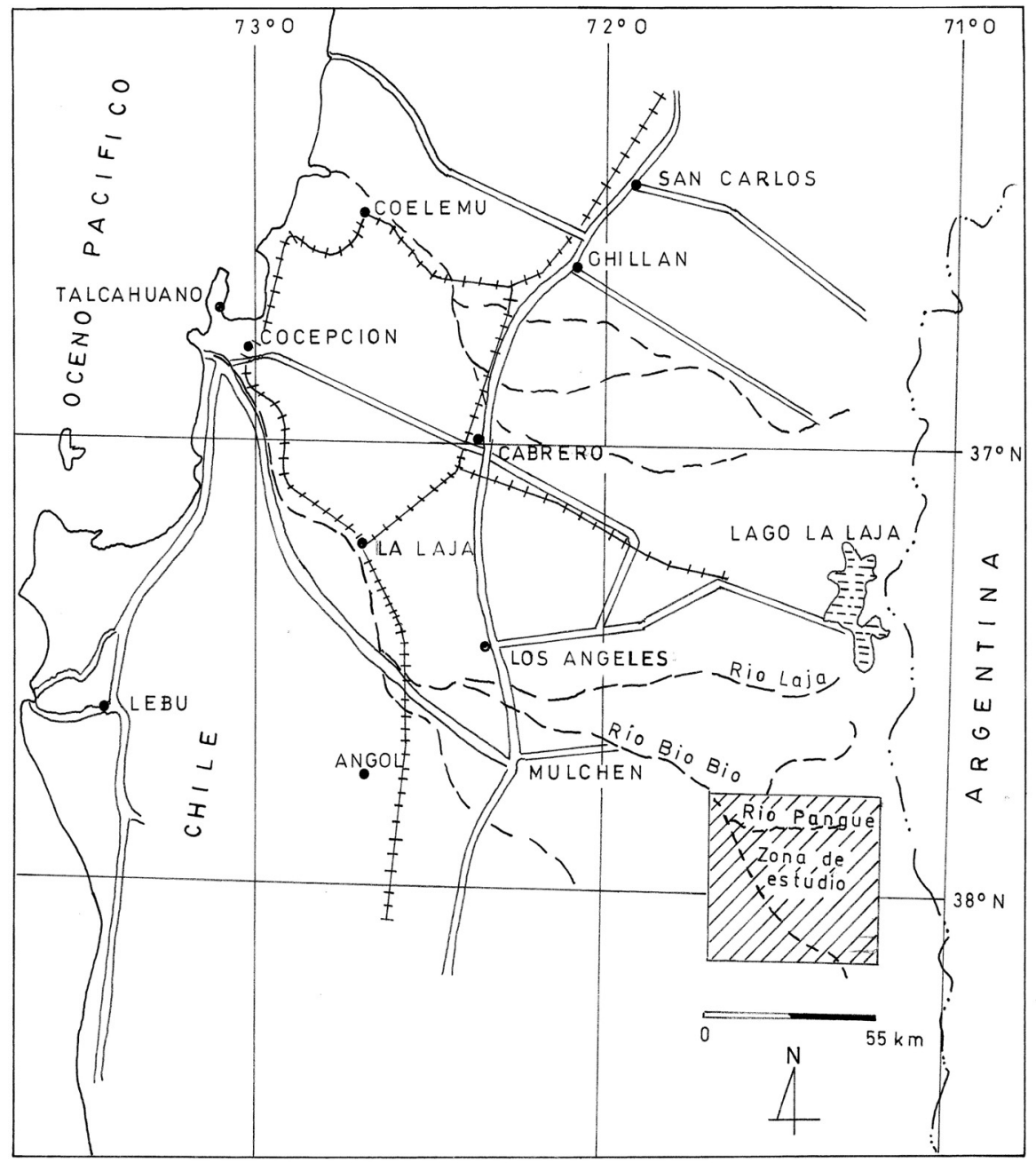

Figura 1. Plano de ubicación de la zona de estudio y sus alrededores.

Para determinar la magnitud de las actividades laháricas que pudiesen formarse durante una eventual erupción es necesario conocer la distribución de los glaciares, su espesor, volumen, extensión y volumen del manto nival invernal.

Finalmente con el objeto de predecir una posible erupción del volcán Callaqui se hace necesario integrar los antecedentes obtenidos (volcán Santa Elena, Lipman y Mullineaux, 1980; Gonzalez-Ferran, 1987; Hicks y Woodward, 1989) con una buena instrumentalización a fin de observar su comportamiento presente y futuro. 


\section{Glaciares del volcán Callaqui}

Un estudio realizado por Marangunic y Moreno (1981) que tuvo como característica evaluar preliminarmente los efectos que el volcán Callaqui podría producir ante una eventual erupción en la central Pangue, permitió realizar un catastro de los glaciares con sus áreas, orientaciones, cotas y su clasificación según las normas de la Organización de las Naciones Unidas para la Educación, la Ciencia y la Cultura (UNESCO, 1993).

Dicho estudio permitió solamente obtener una estimación de espesores comparando su extensión areal con glaciares ubicados en otras regiones, de tal manera que los volúmenes estimados necesitan ser evaluados con mediciones de espesores realizados en terreno.

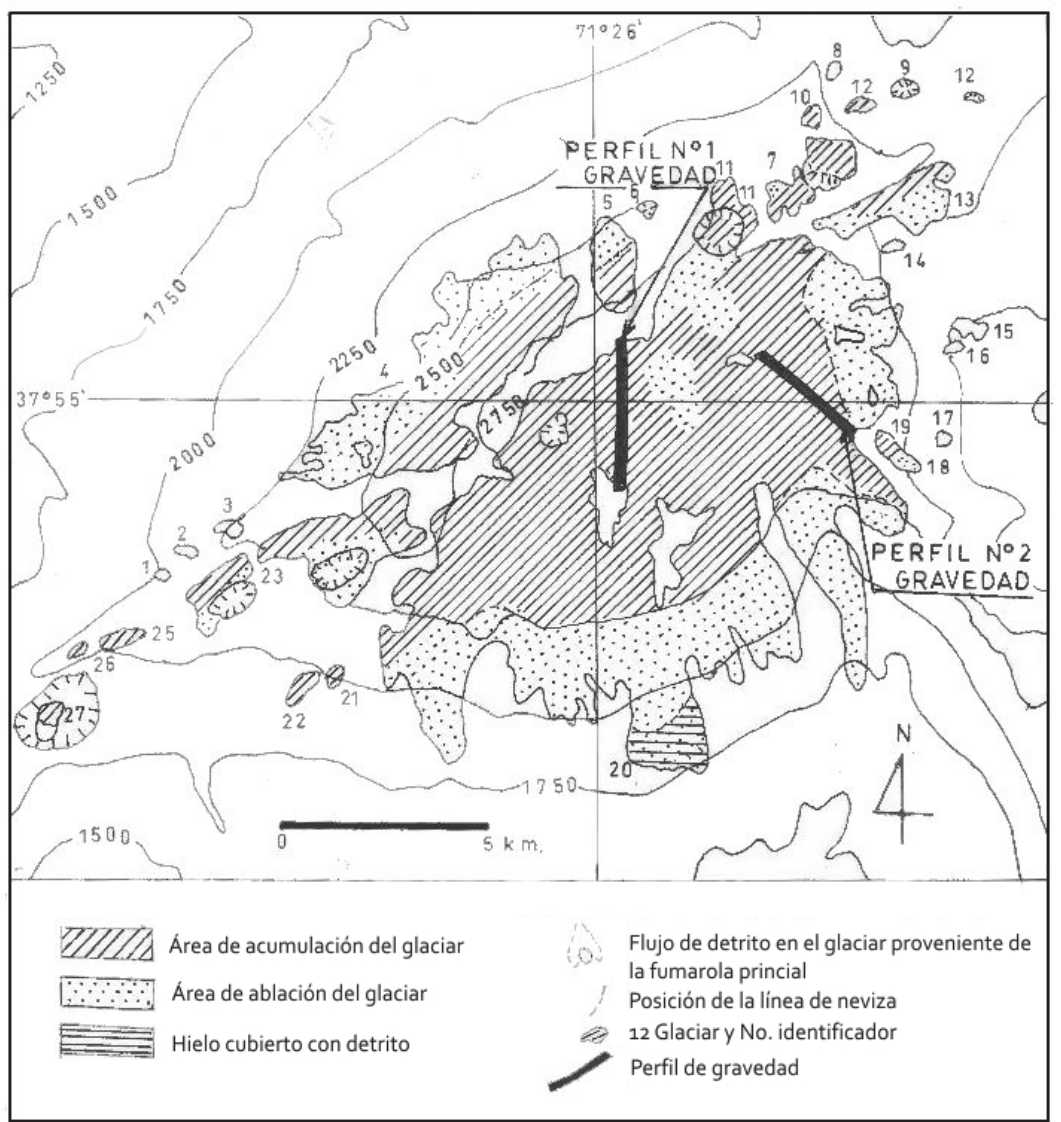

Figura 2. Distribución de los glaciares en el volcán Callaqui, Marangunic y Moreno (1981) y ubicación de perfiles gravimétricos, Araneda (1984). 
Tabla 1

Estimación del volumen de hielo de los glaciares en el área de estudio según nomenclatura de Marangunic y Moreno (1981)

\begin{tabular}{|c|c|c|c|c|}
\hline $\begin{array}{l}\text { Número del } \\
\text { glaciar }\end{array}$ & $\begin{array}{l}\text { Area } \times 10^{-3} \\
\quad\left(\mathrm{~km}^{2}\right)\end{array}$ & $\begin{array}{c}\text { Espesor } \\
\text { medio }(m)\end{array}$ & $\begin{array}{c}\text { Volumen de } \\
\text { hielo } \times 10^{-6}\left(\mathrm{~km}^{3}\right)\end{array}$ & $\begin{array}{c}\text { Volumen de } \\
\text { agua } \times 10^{-6}\left(\mathrm{~km}^{3}\right)\end{array}$ \\
\hline 1 & 3 & 1 & 3 & 2 \\
\hline 2 & 30 & 5 & 150 & 120 \\
\hline 3 & 3 & 1 & 3 & 2 \\
\hline 4 & 1,840 & 46 & 84,640 & 67,712 \\
\hline 5 & 230 & 15 & 3,450 & 2,760 \\
\hline 6 & 4 & 1 & 4 & 3 \\
\hline 7 & 80 & 9 & 720 & 576 \\
\hline 8 & 10 & 3 & 30 & 24 \\
\hline 9 & 12 & 3 & 36 & 29 \\
\hline 10 & 15 & 4 & 60 & 48 \\
\hline 11 & 110 & 10 & 1,100 & 880 \\
\hline 12 & 5 & 1 & 5 & 4 \\
\hline 13 & 330 & 17 & 5,610 & 4,488 \\
\hline 14 & 5 & 1 & 5 & 4 \\
\hline 15 & 3 & 1 & 3 & 2 \\
\hline 16 & 4 & 1 & 4 & 3 \\
\hline 17 & 3 & 1 & 3 & 2 \\
\hline 18 & 3 & 1 & 3 & 2 \\
\hline 19 & 70 & 8 & 560 & 448 \\
\hline 20 & 11,070 & 98 & $1,084,860$ & 867,888 \\
\hline 21 & 12 & 3 & 36 & 29 \\
\hline 22 & 15 & 4 & 60 & 48 \\
\hline 23 & 540 & 24 & 12,960 & 10,368 \\
\hline 24 & 160 & 12 & 1,920 & 1,536 \\
\hline 25 & 40 & 6 & 240 & 192 \\
\hline 26 & 3 & 1 & 3 & 2 \\
\hline 27 & 8 & 2 & 16 & 13 \\
\hline 28 & 7 & 2 & 14 & 11 \\
\hline 29 & 3 & 1 & 3 & 2 \\
\hline 30 & 3 & 1 & 3 & 2 \\
\hline 31 & 3 & 1 & 3 & 2 \\
\hline 32 & 7 & 2 & 14 & 11 \\
\hline Total & 14,631 & & $1,196,521$ & 957,213 \\
\hline
\end{tabular}


El trabajo anteriormente descrito permitió identificar 32 glaciares los cuales unos corresponden a un casquete de hielo de $11,070 \mathrm{~km}^{2}$ y el resto a pequeños glaciares de montaña, glaciares y campos de nieve. El conjunto de glaciares determinados cubre un área de $14,631 \mathrm{~km}^{2}$. La Tabla 1 muestra una estimación del volumen de hielo y sus equivalentes en agua, calculados con una densidad media del hielo de $0.8 \mathrm{gr} / \mathrm{cm}^{3}$, obtenido del estudio de Marangunic y Moreno $(1979,1981)$ (Figura 2).

\section{Determinación de los volúmenes de hielo}

Con el objeto de tener un cuadro más realista del volumen de hielo en el volcán Callaqui se efectuaron dos perfiles gravimétricos para determinar sus respectivos espesores de hielo, ellos fueron ubicados en zonas donde la potencia de hielo, se supone mayor (cráter del volcán). Junto a lo anterior se realizaron reconocimientos aéreos de la zona que, con apoyo fotográfico permitieron cuantificar aquellos glaciares de mayor relevancia.

Este estudio arrojó nuevos datos sobre los actuales glaciares y ha permitido descartar algunos pequeños que no presentan hielos perennes. A fin de uniformar los resultados entregados se utilizó la nomenclatura de Marangunic y Moreno (1981) para designar los distintos glaciares (Tabla 1).

\section{Mediciones gravimétricas}

Los datos fueron obtenidos mediante un gravímetro LaCoste y Romberg modelo $\mathrm{G}$ $\mathrm{N}^{\circ}$ 411. Como estación base se consideraron las estaciones iniciales de cada perfil, debido a las condiciones topográficas imperantes en la zona. De esta forma los valores de la gravedad obtenidos fueron relativos.

La ubicación y cotas de las estaciones gravimétricas se obtuvieron mediante un levantamiento taquimétrico, enlazando las cotas a un punto de altura conocido en el campamento Pangue vía altimetría. Las medidas de gravedad son tomadas en diversas situaciones que las afectan, de modo que para hacerlas comparables entre sí deben ser corregidas.

Las correcciones consideradas fueron: latitud, topográfica, aire libre $(0.3086 \mathrm{mGal} / \mathrm{m}$ altura $)$ y Bouguer. Para esta última se consideró una placa infinita equivalente a hielo de densidad $\rho=0.8 \mathrm{gr} / \mathrm{cm}^{3}$ de tal forma que el valor para esta corrección fue de $0.03352 \mathrm{mGal} / \mathrm{m}$ de altura.

En la Tabla 2 se dan los valores de las estaciones gravimétricas, correcciones y las diferencias relativas de la anomalía de Bouguer para cada una de ellas. 
Tabla 2

Estaciones gravimétricas de los perfiles 1 y 2 con sus respectivas correcciones y diferencias relativas de la anomalía de Bouguer

\begin{tabular}{|c|c|c|c|c|c|c|c|}
\hline Estación & $\begin{array}{c}\text { Cota } \\
\text { (m) }\end{array}$ & $\begin{array}{c}\text { Dif. Relat. } \\
\text { de gravedad } \\
\text { observada } \\
\text { (mGal) }\end{array}$ & $\begin{array}{c}\text { Correc. de } \\
\text { aire libre } \\
\text { (mGal) }\end{array}$ & $\begin{array}{c}\text { Correc. de } \\
\text { Bouguer } \\
\text { (mGal) }\end{array}$ & $\begin{array}{l}\text { Correc. } \\
\text { topográfica } \\
\text { (mGal) }\end{array}$ & $\begin{array}{l}\text { Correc. } \\
\text { de latitud } \\
\text { (mGal) }\end{array}$ & $\begin{array}{c}\text { Dif. Relat. de } \\
\text { anomalía } \\
\text { de Bouguer } \\
\text { (mGal) }\end{array}$ \\
\hline \multicolumn{8}{|c|}{ Perfil 1} \\
\hline 1 & 3010 & 2914.66 & 928.89 & -113.51 & 10.88 & 1.29 & 3742.21 \\
\hline 2 & 3008 & 2915.35 & 928.27 & -113.43 & 10.87 & 1.21 & 3742.27 \\
\hline 3 & 3011 & 1916.05 & 929.19 & -113.54 & 10.86 & 1.12 & 3743.68 \\
\hline 4 & 3034 & 2907.75 & 936.29 & -114.41 & 10.84 & 0.98 & 3741.45 \\
\hline 5 & 3044 & 2904.19 & 939.38 & -114.79 & 10.82 & 0.88 & 3740.48 \\
\hline 6 & 3027 & 2910.77 & 934.13 & -114.15 & 10.80 & 0.79 & 3742.34 \\
\hline \multicolumn{8}{|c|}{ Perfil 2} \\
\hline 1 & 2523 & 3060.11 & 778.60 & -95.14 & 9.00 & 0.91 & 3753.48 \\
\hline 2 & 2522 & 3060.45 & 778.29 & -95.10 & 9.08 & 9.93 & 3753.65 \\
\hline 3 & 2531 & 3058.45 & 781.07 & -95.44 & 9.17 & 0.95 & 3754.20 \\
\hline 4 & 2538 & 3056.53 & 783.23 & -95.71 & 9.27 & 0.99 & 3754.31 \\
\hline 5 & 2556 & 3053.07 & 788.78 & -96.39 & 9.38 & 1.03 & 3755.37 \\
\hline
\end{tabular}

\section{Interpretación de las anomalías}

Debido a la pequeña longitud de los perfiles gravimétricos se consideran gradientes locales para estimar el efecto regional de la zona que restados a los valores de la anomalía de Bouguer da la anomalía residual la cual se interpretó como la producida por el espesor de hielo. Para cuantificar esta anomalía residual se usó el método de Talwani, Talwani, Werzel y Landisman (1959) para cuerpos bidimensionales. Esta interpretación puede realizarse con bastante exactitud debido al gran contraste de densidad $\left(\Delta \rho=1.7 \mathrm{gr} / \mathrm{cm}^{3}\right)$ existentes entre el hielo y la roca basal, $\rho=0.8 \mathrm{gr} / \mathrm{cm}^{3}$ y $\rho=2.5 \mathrm{gr} / \mathrm{cm}^{3}$, respectivamente.

La Tabla 3 muestra un resumen de los resultados obtenidos. Las Figuras $3 \mathrm{a}$ y $3 \mathrm{~b}$ muestra gráficamente los resultados de los perfiles de gravedad 1 y 2 .

\section{Estimación de volúmenes de hielo}

La Tabla 4 contiene la estimación de volúmenes máximos de hielo en los glaciares más relevantes, según la nomenclatura empleada por Marangunic y Moreno (1981). Estos fueron obtenidos mediante el apoyo de fotografías aéreas, vuelos de reconocimientos a baja altura y observaciones directas en terreno.

Los glaciales que no aparecen en el listado, corresponden más bien a acumulaciones de hielos temporales. 
Tabla 3

Muestra los perfiles 1 y 2 de gravedad con la determinación de la anomalía residual, espesor de hielo calculado y cota del lecho rocoso

\begin{tabular}{|c|c|c|c|}
\hline Estación & $\begin{array}{l}\text { Anomalía residual } \\
\text { (mGal) }\end{array}$ & $\begin{array}{c}\text { Espesor de hielo } \\
(\mathrm{m})\end{array}$ & $\begin{array}{c}\text { Cota lecho de roca } \\
(\mathrm{m})\end{array}$ \\
\hline \multicolumn{4}{|c|}{ Perfil 1} \\
\hline 1 & 0.00 & 0 & 3,010 \\
\hline 2 & 0.60 & 11 & 2,997 \\
\hline 3 & 0.00 & 1 & 3,010 \\
\hline 4 & 1.50 & 25 & 3,009 \\
\hline 5 & 2.27 & 37 & 3,007 \\
\hline 6 & 0.00 & 0 & 3,027 \\
\hline \multicolumn{4}{|c|}{ Perfil 2} \\
\hline 1 & 0.00 & 0 & 2,523 \\
\hline 2 & 0.43 & 7 & 2,515 \\
\hline 3 & 0.36 & 6 & 2,525 \\
\hline 4 & 0.66 & 10 & 2,528 \\
\hline 5 & 0.00 & 0 & 2,556 \\
\hline
\end{tabular}
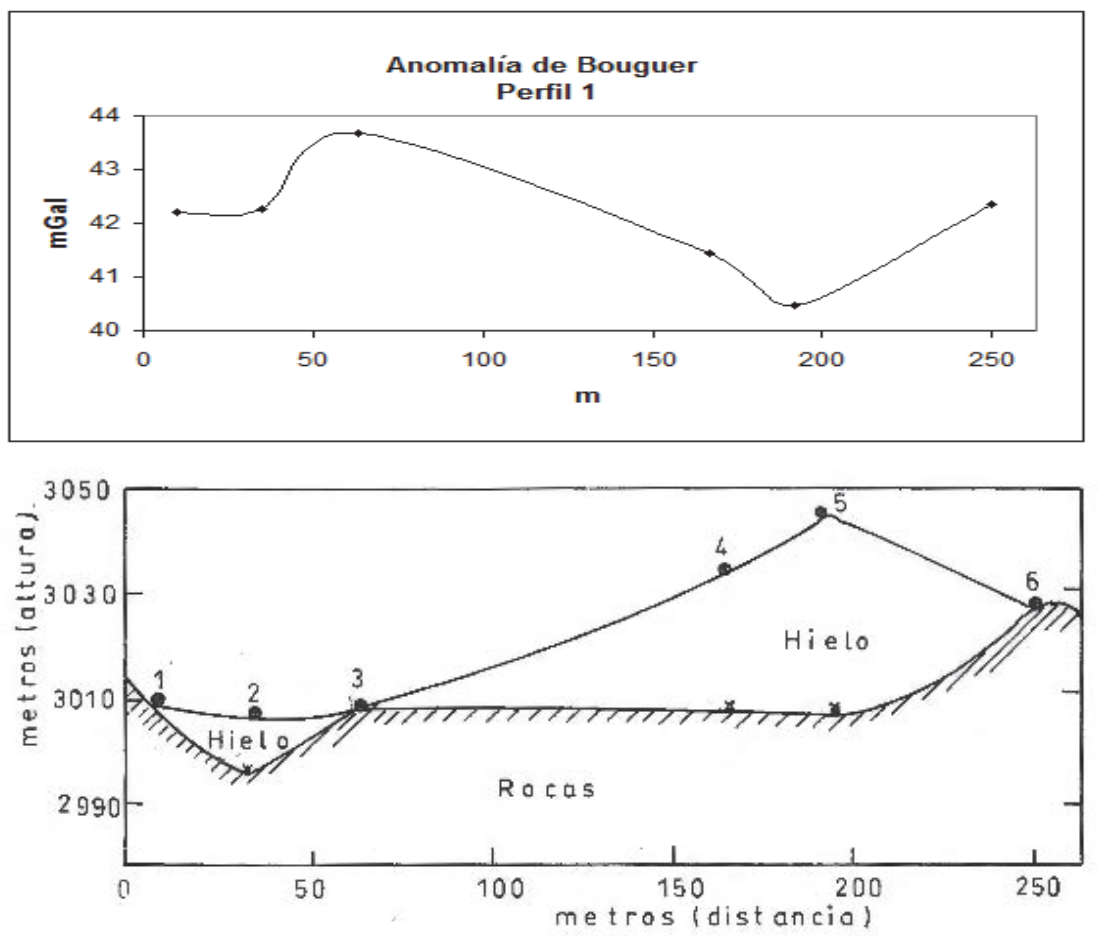

Figura 3a. Interpretación Perfil Gravimétrico 1. 

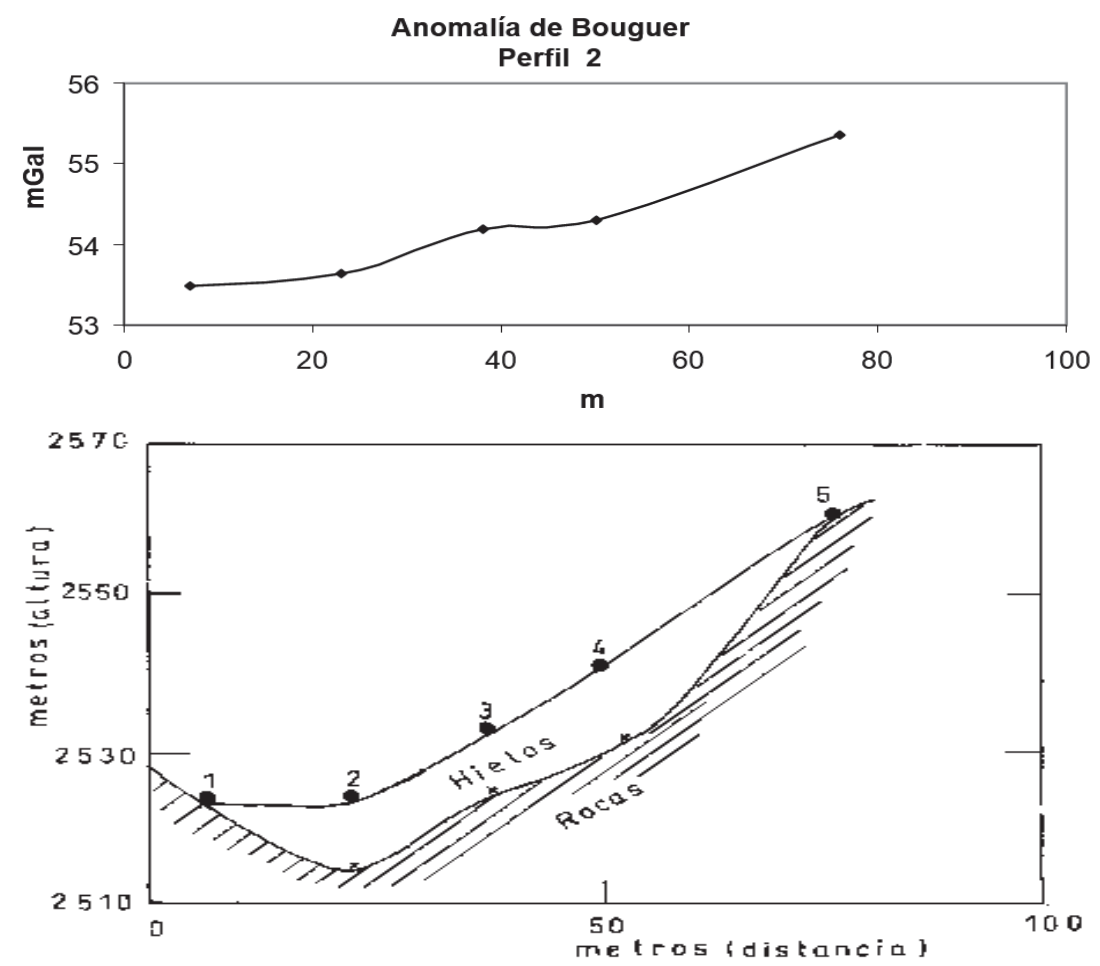

Figura 3b. Interpretación Perfil Gravimétrico 2.

Tabla 4

Muestra una estimación de los volúmenes máximos en los glaciares más relevantes

\begin{tabular}{ccrc}
\hline $\begin{array}{c}\text { Núm. del } \\
\text { glacial }\end{array}$ & $\begin{array}{c}\text { Area } \times 10^{-3} \\
\left(\mathrm{~km}^{2}\right)\end{array}$ & $\begin{array}{r}\text { Espesor medio } \\
(\mathrm{m})\end{array}$ & $\begin{array}{c}\text { Volumen de hielo } \times 10^{-6} \\
\left(\mathrm{~km}^{3}\right)\end{array}$ \\
\hline 2 & 30 & 3 & 9 \\
4 & 1,540 & 15 & 23,100 \\
5 & 150 & 8 & 1,200 \\
7 & 250 & 6 & 1,500 \\
8 & 10 & 2 & 20 \\
9 & 12 & 2 & 24 \\
10 & 15 & 3 & 45 \\
11 & 200 & 8 & 1,600 \\
13 & 300 & 15 & 1,500 \\
19 & 70 & 8 & 560 \\
20 & 11,070 & 6 & 3,000 \\
23 & 500 & 6 & 3,000 \\
24 & 160 & 6 & 960 \\
25 & 40 & 5 & 200 \\
\hline Total & 14,347 & & 424,168 \\
\hline
\end{tabular}


Es importante señalar que la cubierta nival estacional aporta un volumen adicional que es muy variable año a año, especialmente entre los meses de mayo a diciembre. No obstante, en un año normal de precipitaciones se genera un manto de nieve invernal sobre la cota de $1,500 \mathrm{msnm}$ cuyo espesor promedio se estima en $3 \mathrm{~m}$. Este manto permanece en los flancos del volcán entre junio y octubre.

Para determinar los volúmenes de agua equivalente a un volumen determinado de hielo, se estima una densidad media del hielo de $0.8 \mathrm{gr} / \mathrm{cm}^{3}$, considerando la presencia de grietas, impurezas, burbujas de aire, baja densidad cerca de la superficie, etc.

El volumen total de hielo en los glaciares analizados del volcán Callaqui, corresponden a $0.42168 \mathrm{~km}^{3}$ equivalentes, en consecuencia, a un volumen de $0.339334 \mathrm{~km}^{3}$ de agua.

Por otra parte, la nieve tiene una densidad mucho menor que la del hielo y ha sido estimada en $0.25 \mathrm{gr} / \mathrm{cm}^{3}$.

\section{Sistemas de monitereo en el volcán Callaqui}

En general los antecedentes considerados en el monitoreo pre-erupción del volcán se dividieron en tres:

a) Lugar: debe tenerse una idea clara de la zona donde se ubica el centro o los centros emisores de un volcán y no considerar solamente el volcán principal.

b) Tiempo: significa que debe determinarse un periodo de tiempo razonable corto en el cual se espera la erupción. Con esto se podrá estructurar un mayor cuadro predictivo.

c) Tipo y magnitud de la erupción: para este efecto deberá considerarse el tamaño de la erupción.

Generalmente estos son los parámetros más difíciles de obtener ya que es cierto reconocer precursores que necesariamente reflejen el tipo y magnitud de una erupción futura. Una predicción ideal sería incluir cada paso del comportamiento del proceso de la erupción.

Los antecedentes expuestos anteriormente nos hacen establecer dos etapas de la predicción dependiendo de la clase de datos obtenidos en el tiempo:

a) Predicciones en un periodo relativamente largo, basado preliminarmente en medidas geodésicas y gravimétricas.

b) Predicciones en un periodo relativamente corto, basado preliminarmente en datos sísmicos obtenidos a través de una red telemétrica y datos de inclinaciones obtenidos a través de la red correspondiente. Estas medidas son sensiblemente importantes dentro de uno a dos días antes de la erupción. 
Las dos etapas mencionadas anteriormente se pueden sobreponer en un periodo de tiempo de dos a cuatro días, cuando los datos sísmicos y de deformaciones son de similar importancia.

Existen otros parámetros que se pueden cuantificar antes de una erupción, pero su comportamiento se presenta irregularmente para un mismo volcán. Esta irregularidad fue notada en las emisiones de $\mathrm{SO}_{2}$ (dióxido de azufre), $\mathrm{CO}_{2}$ (bióxido de carbono) y otros gases en el Monte Santa Elena durante los años 1980 a1981 en los cuales hizo erupción (Casadevall, 1983).

En todo caso, cualquier estudio predictivo tiene como base las probabilidades, pero si existen conclusiones que se basan en causas razonables, es posible que éstas sean más correctas que predicciones puramente probabilísticas basadas solamente en algún patrón determinado.

En general podemos reconocer dos tipos de predicciones en los volcanes, unos basados en la proyección geológica del pasado al presente y otra basada en registros geofísicos tomados en forma sistemática en el tiempo. Con la primera podría llegarse a establecer solamente el tipo de la erupción que podría originarse en un volcán de acuerdo a su historial. Con el segundo tipo de predicción se puede llegar a conocer muy de cerca el momento mismo de la erupción o el comportamiento anómalo que se puede producir en un determinado tiempo.

Lo ideal sería realizar todos los estudios y controles necesarios que nos pueden conducir a pronosticar una erupción o a conocer con el máximo detalle algún comportamiento anómalo de un volcán, de tal manera que ayude a tomar las providencias necesarias, Chadwick et al. (1983), Dzurisin, Westphal, Jonson (1983).

Los volcanes chilenos activos podemos considerarlos, en forma restringida, en dos grupos de acuerdo a su ubicación: volcanes que no presentan grandes riesgos ante una erupción y volcanes que presentan riesgo de consideración ante una erupción (pérdidas de vida, destrucción de obras civiles, campos de cultivo, fauna, etc.) (Figura 4).

El volcán Callaqui se puede considerar dentro del segundo grupo, por lo tanto su instrumentalización para monitorear su actividad se hace necesaria. Considerando la topografía accidentada de la zona y al gran apoyo logístico que se requiere para obtener un set de datos sistemáticos en el tiempo, se ha creído conveniente indicar solamente aquellos sistemas que presentan una mayor ventaja en la obtención de datos y que se encuadran dentro de las predicciones de periodos relativamente largos y periodos relativamente cortos. Dentro de las primeras podrían instalarse una serie de pilares materializados alrededor del edificio volcánico a diferentes alturas. Estos debieran poseer un vástago de acero en su centro, de tal manera que pudiese medirse su variación horizontal como vertical con un control geodésico de alta precisión. 


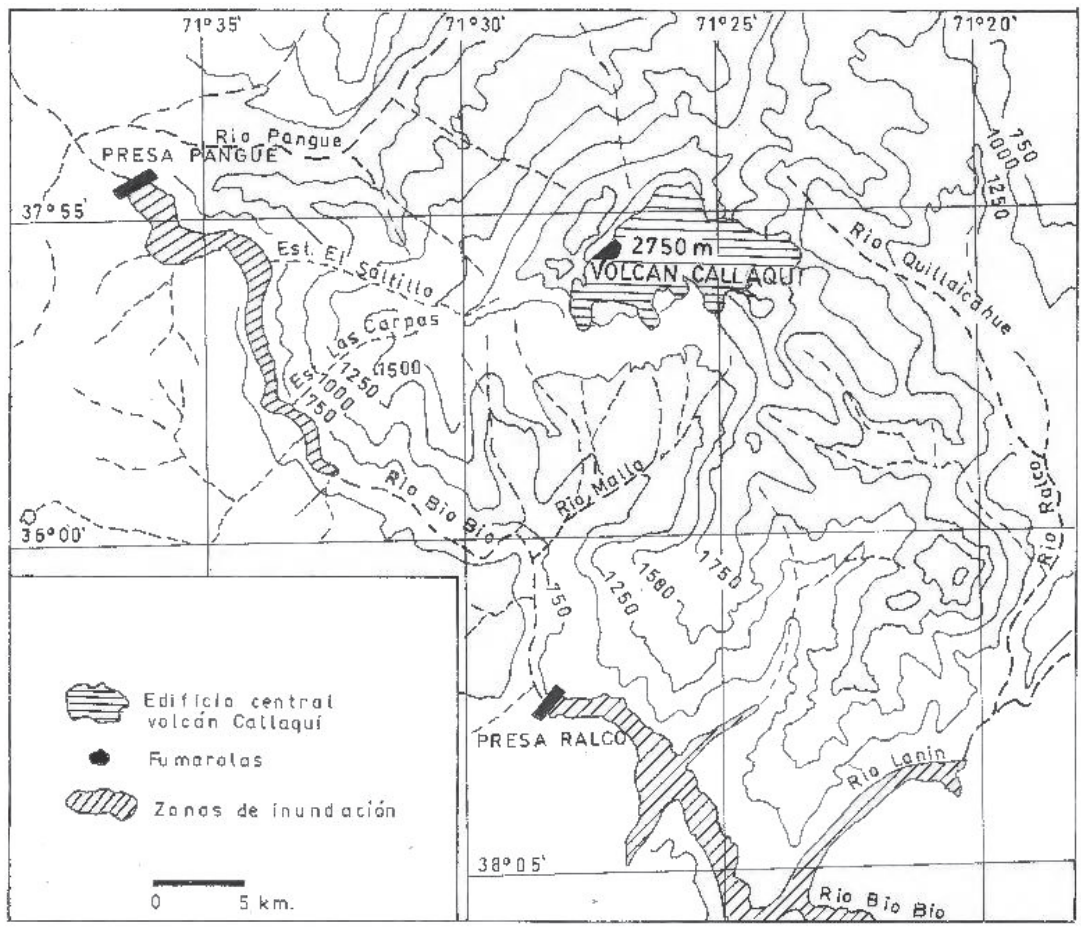

Figura 4. Cuencas más relevantes del complejo del volcán Callaqui.

En las predicciones de periodos relativamente cortos, es conveniente instalar una red sísmica telemétrica compuesta al menos por cuatro sismógrafos, en la cual el centro de recopilación de la información podría estar en la central Puangue o Ralco. La ubicación de las estaciones (sismógrafos) debería quedar equidistante entre sí, permitiendo detectar con precisión los hipocentros relacionados de alguna forma con el flujo magmático. También dentro de este tipo de predicciones debe contemplarse una red de inclinómetros instalados en el cono volcánico con el objeto de observar deformaciones laterales expresadas en ángulos. El número de ellos será condicionado especialmente a recursos disponibles.

\section{Conclusiones}

Además de las masas de hielo perenne involucradas ante una posible erupción del volcán Callaqui, éstas se verían incrementadas con flujos de lavas, caídas de piroclásticos y avalanchas laháricas que de alguna manera afectarían a dichas centrales. La extensión afectada podría ser significativamente importante, de tal forma que, los daños serían de difícil cuantificación. 
Es importante destacar que a través de observaciones de fotografías aéreas tomadas en diferentes años, 1980 y 1984 aproximadamente, se puede comprobar un retroceso de aquellos glaciares más relevantes que componen el edificio volcánico Callaqui. Este fenómeno tiene gran importancia en evaluaciones futuras ya que produciría una disminución de los volúmenes de hielo a través del tiempo y un menor riesgo de grandes avalanchas laháricas.

Los volúmenes estimados de material involucrado ante una posible erupción del volcán estudiado, afectarían a estas centrales de la siguiente forma:

a) Central Ralco. Podría ser afectada por lahares con volúmenes máximos estimados de 50 millones de $\mathrm{m}^{3} \mathrm{y}$ caída de pirocláticos finos.

b) Central Pangue. Podría ser afectada por flujos de lavas cuyos volúmenes estimados no excedería de $24 \times 106 \mathrm{~m}^{3}$, lahares con volúmenes de hasta $16 \times 105 \mathrm{~m}^{3}$ y caída de piroclásticos finos.

Con la finalidad de aminorar un desastre de gran envergadura, es conveniente tomar las providencias del caso.

Para este efecto se recomienda estudiar el comportamiento del volcán con el monitoreo, principalmente a través de una red sísmica telemétrica, una red de inclinómetros y una red de pilares, en los cuales se pueda observar movimientos verticales y laterales. La ubicación de cada uno de los instrumentos se planificaría con el objeto de obtener la mejor información.

Cualquier conocimiento que se tenga de las estructuras volcánicas y su posible evaluación del riesgo es importante para un país como Chile ubicado al pie del macizo andino, uno de los más activos de la tierra.

\section{Agradecimientos}

El trabajo presentado corresponde a una parte del estudio del volcán Callaqui relacionado con la geología y riesgo volcánico patrocinado en parte por la empresa ENDESA.

\section{Referencias}

Araneda, M., 1984. “Contribución al estudio del riesgo volcánico del volcán Callaqui”, informe inédito, ENDESA.

Araneda, M., Avendaño, M.S., 1983. “Anomalías gravimétricas en áreas volcánicas, monumentación en el volcán Villarrica", Revista Académica, número extraordinario, Universidad de la Frontera, Temuco, Chile, Jornadas Nacionales de Física, pp. 68-72. 
Casadevall, T., et al, 1983. "Gas Emissions and the Eruption of Mount St. Helens through 1982”, Science, 221(4618).

Chadwick, W., W. Jr. et al., 1983. "Deformation Monitoring at Mount St. Hellens 1981 and 1982", Science, 221(4618).

Dzurisin, D., Westphal, O.J., Johnson, 1983. "Eruption Prediction aided by Electronic Tilmeter Data at Mount St. Helens", Science, 221(4618).

Gonzalez-Ferran, O., 1987. "Evolución geológica de las islas chilenas en el Océano Pacífico", Islas Oceánicas Chilenas, editor J.C. Castilla, Universidad Católica de Chile, pp. 37-44.

Hicks, S.R. and Woodward, D.J., 1989. "Gravity models of the Wairarapa region, New Zealand", NZ Journal of Geology and Geophysics, 21(5), pp. 539-534.

Lipman, P.W. and Mullineaux, D.R., 1980. "The 1980 Eruption of Mount St. Helens", Geological Survey Professional paper 1250, Washington,

Marangunic, C., Moreno, H., 1979. "Effects of a Landslide on Glacier Alaska", Report of Polar Studies, Report No. 30.

Marangunic, C., Moreno, H., 1981. "Posibles efectos del volcán Callaqui en obras de la central Pangue", informe inédito, ENDESA.

Moreno, H., Thiele, R., Lahsen, A., Varela, J., López, L., Araneda, M., Vergara, M., Tavera, J., 1984. "Estudio del volcán Callaqui: Geología y riesgo volcánico", informe inédito, ENDESA.

Talwani, M., Werzel, J.L. and Landisman, M., 1959. "Rapid gravity computation for two dimensional bodies with applications to Mendocino submarine fracture zone", J.G.R., vol. 64.

UNESCO, 1993. Final Report, Regional Office for Science and Technology for South (Indonesia), Fhilippine Institute Volcanology and Seismology, Department of Sience and Technology, $66 \mathrm{pp}$. 